\title{
Clinician Response to Patient Emotion: Impact on Subsequent Communication and Visit Length
}

Mary Catherine Beach, MD, $\mathrm{MPH}^{1,2,3,4}$

Jenny Park ${ }^{1}$

Dingfen Han, PbD ${ }^{1}$

Cbristopher Evans, $M D^{5}$

Richard D. Moore, MD, MHS

Somnath Saba, MD, MPH ${ }^{5,6}$

'Division of General Internal Medicine, Department of Medicine, Johns Hopkins School of Medicine, Baltimore, Maryland

${ }^{2}$ Berman Institute of Bioethics, Johns Hopkins University, Baltimore, Maryland

${ }^{3}$ Department of Health, Behavior \& Society, Bloomberg School of Public Health, Johns Hopkins University, Baltimore, Maryland

${ }^{4}$ Welch Center for Prevention, Epidemiology and Clinical Research, Johns Hopkins University, Baltimore, Maryland

${ }^{5}$ Division of General Internal Medicine and Geriatrics, Oregon Health and Science University, Portland, Oregon

${ }^{6}$ Center to Improve Veteran Involvement in Care, VA Portland Health Care System Portland, Oregon

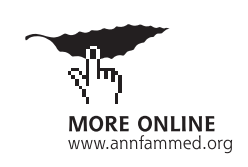

Conflicts of interest: authors report none.

\section{CORRESPONDING AUTHOR}

Mary Catherine Beach Johns Hopkins University 2024 E Monument St, Rm 2-511 Baltimore, MD 21287 mcbeach@jhmi.edu

\begin{abstract}
PURPOSE It is widely cited-based on limited evidence-that attending to a patient's emotions results in shorter visits because patients are less likely to repeat themselves if they feel understood. We evaluated the association of clinician responses to patient emotions with subsequent communication and visit length.
\end{abstract}

METHODS We audio-recorded 41 clinicians with 342 unique patients and used the Verona Coding Definitions of Emotional Sequences (VR-CoDES) to time stamp patient emotional expressions and categorize clinician responses. We used random-intercept multilevel-regression models to evaluate the associations of clinician responses with timing of the expressed emotion, patient repetition, and subsequent length of visit.

RESULTS The mean visit length was 30.4 minutes, with 1,028 emotional expressions total. The majority of clinician responses provided space for the patient to elaborate on the emotion (81\%) and were nonexplicit (56\%). As each minute passed, clinicians had lower odds of providing space (odds ratio $[\mathrm{OR}]=0.96$; $95 \% \mathrm{Cl}, 0.95-0.98)$ and higher odds of being explicit $(\mathrm{OR}=1.02 ; 95 \% \mathrm{Cl}, 1.00$ 1.03). Emotions were more likely to be repeated when clinicians provided space $(\mathrm{OR}=2.33 ; 95 \% \mathrm{Cl}, 1.66-3.27)$, and less likely to be repeated when clinicians were explicit $(\mathrm{OR}=0.61 ; 95 \% \mathrm{Cl}, 0.47-0.80)$. Visits were shorter $(\beta=-0.98$ minutes; $95 \% \mathrm{Cl},-2.19$ to 0.23$)$ when clinicians' responses explicitly focused on patient affect.

CONCLUSIONS If saving time is a goal, clinicians should consider responses that explicitly address a patient's emotion. Arguments for providing space for patients to discuss emotional issues should focus on other benefits, including patients' well-being.

Ann Fam Med 2021;19:515-520. https://doi.org/10.1370/afm.2740.

\section{INTRODUCTION}

linician empathy in health care settings has been defined as a communication behavior "that demonstrates an accurate understanding and acceptance of the patient's feelings or concerns,"1 and it is generally believed that providing empathy in response to patient emotions is an important part of the clinician's therapeutic response. ${ }^{2,3}$ Several communication coding systems have facilitated the study of emotional communication; these systems typically involve procedures for identifying and coding emotional expressions made by the patient (an empathic opportunity) and then coding different types of clinician response. ${ }^{4-6}$ Using these methods, studies have repeatedly demonstrated that clinicians miss most opportunities to express empathy in response to patient emotional expressions. ${ }^{7.8}$

One hypothesis for why clinicians do not make explicit empathy statements is that they may not feel they have enough time to address their patient's emotions in addition to all the other competing concerns. To alleviate clinicians' concerns about the time involved in responding to emotions, it is argued that providing empathic responses to patients saves time. The evidence for this claim is based on a 1997 study of 116 patient-clinician encounters, which discovered that visits with missed opportunities 
to acknowledge patients' feelings averaged 3 minutes longer than visits with a more positive response. ${ }^{8}$ The same study also found that in $53 \%$ of visits with missed opportunities, the patient brought up the same concern more than once. This finding suggests that patients who have not had their emotional state adequately acknowledged and do not perceive being heard by their clinician may feel compelled to repeat themselves.

The conclusions from this landmark study are appealing for anyone who believes that expressions of empathy are important, particularly for those who teach communication, yet it has never been replicated. Knowing the impact of clinician communication behaviors on visit length is highly relevant for clinicians, who have to make choices at each moment in each encounter about how to spend a fixed amount of time in the best interests of their patients. We therefore designed this study to evaluate the relationship between the clinician's response to a patient's emotion and subsequent communication, including the patient's repetition of emotional expressions and length of visit, using a comprehensive, detailed emotional communication coding system.

\section{METHODS}

\section{Study Design, Participants, and Setting}

We conducted an observational analysis of patient-clinician communication during audio-recorded encounters collected as part of the MaRIPOHSA (Maximizing Respect and Improving Patient Outcomes in HIV and Substance Abuse) Study. The MaRIPOHSA Study took place at the HIV specialty clinics in 2 urban academic medical centers and was approved by their institutional review boards. Although the data were collected in HIV specialty clinics, it is important to note that many patients in these clinics obtain their primary care from their HIV clinician. Clinicians (physicians, nurse practitioners, and physician assistants) were recruited at staff meetings and were eligible to enroll if they provided primary care to patients with HIV. A convenience sample of adult patients of participating clinicians were recruited from waiting rooms. Patients were eligible to enroll if they were established in HIV care for at least 6 months, were being seen by their regular HIV clinician for a routine visit, and were planning to speak English. The only exclusion criteria for patients were not being able to understand or give informed consent. All participants (clinicians, patients, and any third-party companions) provided written informed consent before any research procedure.

\section{Data Collection}

We placed 2 audio recorders in the examination room immediately before the encounter. A professional company transcribed the audio recordings, and indicated timestamps (eg, "54 seconds") next to each line of dialog. Research assistants checked the transcripts for accuracy. Patients and clinicians completed brief questionnaires.

\section{Coding of Emotional Communication}

We coded the transcripts using the Verona Coding Definitions of Emotional Sequences (VR-CoDES), which has been used in multiple settings to describe emotional communication between the patient and clinician ${ }^{5,6}$ with high reliability and validity. ${ }^{9,10}$ All transcripts for this study were coded by the same investigator (J.P.) who trained using the VR-CoDES training manuals and had extensive previous experience.

\section{Patient Emotional Expressions}

The VR-CoDES categorizes patient emotional expressions into concerns and cues according to the level of intensity. A concern is defined as "a clear and unambiguous expression of an unpleasant current or recent emotion where the emotion is explicitly verbalized," and a cue is defined as "a hint which suggests an underlying unpleasant emotion." ${ }^{15}$ We further coded emotional expressions as either initial (the first time the patient raises the emotional situation) or subsequent (repetition of an emotional situation stated previously). For example, if a patient brought up losing their job several times, each reference to job loss after the first would count as a repetition. On the other hand, if the patient brought up losing their job and then also brought up being worried about their recent computed tomography scan, these would count as different emotional expressions.

\section{Clinician Response to Patient Emotional Expression} VR-CoDES classifies clinician responses into 1 of 17 mutually exclusive categories, and a detailed description of these categories is provided in the original published study. ${ }^{6}$ These response categories are broadly grouped according to 2 primary features-whether the clinician refers explicitly to the content or emotion of the emotional issue (explicit vs nonexplicit), and whether the clinician provides the patient the opportunity to express their negative emotions further (provides vs reduces space). ${ }^{6}$ We further grouped clinician responses that reduced space into 2 subcategories based on whether the clinician gave information or advice, or tried to block further discussion of the emotional expression. We further grouped responses that provided space into 5 subcategories depending on whether the clinician expressed empathy (a verbal expression that shows clinician understanding of patient emotion), focused explicitly on the patient's emotion, explored 
the emotional issue, provided acknowledgment, or gave a passive response (silence, back-channeling, or nonexplicit acknowledgment). The reorganizations of the original codes have been used in previous studies. ${ }^{11-13}$

\section{Statistical Analysis}

All analyses were done using Stata statistical software, version 16 (StataCorp LLC). We used descriptive statistics to explore patient, clinician, visit, and emotional communication characteristics. We conducted multilevel mixed-effects logistic regression analysis to assess odds of each clinician response type (dependent variable) according to the timestamp of the emotional expression (independent variable). Because some research suggests that patients whose emotions have not been adequately addressed are more likely to repeat the emotion, we also conducted multilevel mixed-effects logistic regression analysis to assess the odds of a patient repeating the emotional expression (dependent variable) according to each type of clinician response (independent variables). Finally, we used multilevel mixed-effects linear regression analysis to assess associations between types of clinician responses (independent variables) and subsequent length of visit (elapsed time between emotional expression and the end of the visit). To account for potential confounding of associations of response types with subsequent length of visit by the tendency of clinicians to respond differently as time progressed throughout the visit, we examined the associations between clinician responses (independent variable) and visit length (dependent variable) while adjusting for the timestamp of the emotional expression. Because each visit could have more than one emotional expression and response, the subsequent length of visit was different for each individual emotional expression-response pair. All regression analyses (unadjusted and adjusted) accounted for nesting of patient emotional expressions within each visit and nesting of patient visits within clinicians. Finally, we repeated all regression analyses adjusting for clinician and patient age, sex, and race/ethnicity.

\section{RESULTS}

\section{Sample Characteristics}

Most eligible clinicians (89\%) and eligible patients (92\%) enrolled in the study. Supplemental Table 1, available at https://www.AnnFamMed.org/lookup/ suppl/doi:10.1370/afm.2740/-/DC1, shows the sample characteristics. Clinicians were predominantly female (66\%) and White (66\%). The majority of patients were male (64\%) and African American (77\%). A small number of patient visits (5\%) included a companion, and $20 \%$ involved a second clinician (eg, nurse or resident).
The average encounter length was 30.4 minutes, and two-thirds of visits (67\%) had at least 1 emotional expression. Within the 342 encounters, there were 1,028 emotional expressions. About one-half (51\%) of the emotional expressions were initial, while the rest (49\%) were subsequent (repetitions of previously stated emotions). Emotional expressions occurred throughout the encounter, with a mean (SD) timestamp of 10.1 (9.2) minutes, and a range from 0.05 minutes to 40.6 minutes.

\section{Clinician Responses to Patient Emotional Expressions}

The large majority of clinician responses (81\%) broadly provided space for the patient to elaborate on the emotion, and more than one-half of responses (56\%) were nonexplicit. Supplemental Table 2, available at https:// www.AnnFamMed.org/lookup/suppl/doi:10.1370/ afm.2740/-/DC1, shows the frequencies of each specific type of clinician response. The most common specific clinician response was categorized as neutral/ passive (eg, back-channeling). The next most common responses were those that explored the emotional expression by asking questions (22\%), acknowledging the emotion/circumstance (20\%), or giving information/advice (12\%). Responses categorized as empathy were uncommon $(5 \%)$, as were those that blocked the patient from talking more about the emotion (6\%).

\section{Association of Emotional Expression Timing With Clinician Response}

Supplemental Table 3, available at https://www.Ann FamMed.org/lookup/suppl/doi:10.1370/afm.2740/-/ DC1, shows the odds of each clinician response type according to the timestamp of the patient emotional expression. As each minute of the visit passed, the odds of a clinician providing space for the patient to elaborate about an emotional expression decreased significantly by $4 \%$, and the odds of a clinician being explicit increased significantly by $2 \%$. The diminishing likelihood of providing space may be a result of both decreased odds of a neutral/passive response and increased odds of both giving information/advice and blocking the discussion. Additional adjustment for clinician and patient age, sex, and race/ethnicity did not change these results.

\section{Association of Clinician Response Type With Patient Repetition of Emotional Expression}

Patients had significantly greater odds of repeating their emotions when given vs not given space (odds ratio $[\mathrm{OR}]=2.33 ; 95 \% \mathrm{CI}, 1.66-3.27$ ) and significantly reduced odds of repeating their emotions when clinicians responded explicitly vs nonexplicitly $(\mathrm{OR}=0.61 ; 95 \% \mathrm{CI}, 0.47-0.80)$. This result seemed to 
be accounted for primarily by higher odds of repeating emotions after a neutral/passive response $(\mathrm{OR}=1.95$; 95\% CI, 1.50-2.54) and lower odds of repeating emotions after both types of space-reducing responses $(\mathrm{OR}=0.50 ; 95 \% \mathrm{CI}, 0.33-0.75$ for information/advice $\mathrm{OR}=0.36 ; 95 \% \mathrm{CI}, 0.20-0.64$ for blocking). There was no association with clinician responses categorized as empathy. Additional adjustment for clinician and patient age, sex, and race/ethnicity did not change these results.

\section{Association of Clinician Response Type With Visit Length}

Table 1 displays differences in subsequent length of visit after each type of clinician response. Clinician responses that provided (vs reduced) space were associated with a significantly longer subsequent length of visit in unadjusted analyses (2.95 minutes longer; 95\% CI, 1.55-4.35) as well as in analyses that accounted for clinicians' tendency to respond with less space as time passed (1.75 minutes longer; $95 \%$ CI, 0.20 3.29). After additional adjustment for clinician and patient age, sex, and race/ethnicity, this finding was not significant $(P=.19)$. Clinician responses that give information/advice were associated with shorter visits both in unadjusted analyses (3.51 minutes shorter; 95\% $\mathrm{CI},-5.20$ to -1.82$)$ and in analyses that accounted for clinicians' tendency to give more information as time passed (1.89 minutes shorter; 95\% CI, -3.74 to -0.04 ); however, this result was no longer significant after adjusting for clinician and patient age, sex, and race/ ethnicity $(P=.17)$.

Explicit (vs nonexplicit) clinician responses were associated with a shorter subsequent length of visit (1.20 minutes shorter; $95 \% \mathrm{CI},-2.30$ to -0.10$)$ in unadjusted analyses, however this association was not significant after adjustment for the tendency of clinicians to make more explicit responses as time passed in the visit as well as clinician and patient age, sex, and race/ethnicity $(P=.08)$. On the other hand, responses that explicitly focused on patient affect were associated with shorter visits in fully adjusted analyses (4.11 minutes shorter; $95 \% \mathrm{CI},-8.0$ to -0.21$)$. There was no association of clinician responses categorized as empathy with visit length.

\section{DISCUSSION}

Emotional communication may have a more complicated relationship with visit length than previously thought. We found that clinicians vary in how they respond to patient emotions as time passes during visits, and that empathic statements are rare and not associated with visit length. Our results add nuance to the interpretation of an earlier landmark study by Levinson et $\mathrm{al}^{8}$ demonstrating the timesaving nature of empathic emotional responses. We found a trend suggesting that providing space for patients to elaborate

Table 1. Association of Clinician Response With Subsequent Length of Visit

\begin{tabular}{|c|c|c|c|}
\hline \multirow[b]{2}{*}{ Type of Clinician Response } & \multicolumn{3}{|c|}{ Subsequent Length of Visit, $\beta(95 \% \mathrm{Cl})$, Minutes } \\
\hline & Unadjusted Modela & Model $1^{b}$ & Model $2^{c}$ \\
\hline \multicolumn{4}{|l|}{ Broad response } \\
\hline Explicit vs nonexplicit & $-1.20(-2.30$ to -0.10$)$ & $-0.98(-2.19$ to 0.23$)$ & $-1.37(-2.89$ to 0.15$)$ \\
\hline Provides vs reduces space & 2.95 (1.55 to 4.35$)$ & $1.75(0.20$ to 3.29$)$ & $1.27(-0.63$ to 3.18$)$ \\
\hline \multicolumn{4}{|l|}{ Specific response } \\
\hline \multicolumn{4}{|l|}{ Provides space } \\
\hline Empathy & $1.53(-0.99$ to 4.05$)$ & $1.05(-1.77$ to 3.86$)$ & $1.38(-2.26$ to 5.01$)$ \\
\hline Explicit focus on affect & $-0.47(-3.35$ to 2.41$)$ & $-3.06(-6.22$ to 0.10$)$ & $-4.11(-8.00$ to -0.21$)$ \\
\hline Acknowledgment & $-2.02(-3.38$ to -0.65$)$ & $-1.17(-2.69$ to 0.34$)$ & $-1.14(-3.06$ to 0.78$)$ \\
\hline Exploring & $1.37(0.06$ to 2.68$)$ & $1.03(-0.42$ to 2.48$)$ & $0.77(-1.00$ to 2.55$)$ \\
\hline Neutral/passive & $1.56(0.43$ to 2.68$)$ & $0.76(-0.47$ to 2.00$)$ & $0.68(-0.90$ to 2.26$)$ \\
\hline \multicolumn{4}{|l|}{ Reduces space } \\
\hline Gives information/advice & $-3.51(-5.20$ to -1.82$)$ & $-1.89(-3.74$ to -0.04$)$ & $-1.64(-3.96$ to 0.68$)$ \\
\hline Blocking & $-1.75(-3.98$ to 0.48$)$ & $-0.62(-3.06$ to 1.82$)$ & $-0.03(-2.85$ to 2.79$)$ \\
\hline \multicolumn{4}{|c|}{ Note: Values in bold are statistically significant $(P<.05)$. } \\
\hline \multicolumn{4}{|c|}{$\begin{array}{l}\text { a Random intercept univariate multilevel linear regression models (account for clustering of emotional expressions within patient encounters and of patient encounters } \\
\text { within clinicians). } \\
\text { b Random intercept multilevel linear regression models (account for clustering of emotional expressions within clinicians) with adjustment for the timestamp of the } \\
\text { emotional expression. } \\
\text { 'Random intercept multilevel linear regression models (account for clustering of emotional expressions within clinicians) with adjustment for the timestamp of the emo- } \\
\text { tional expression; clinician age, sex, and race/ethnicity; and patient age, sex, and race/ethnicity. }\end{array}$} \\
\hline
\end{tabular}


could be associated with longer visit length. We also found, however, that responses explicitly focusing on patient affect were associated with shorter visit length, which perhaps provides a patient-centered solution for acknowledging emotion without necessarily taking more time and is of practical utility when teaching about communication skills.

The comparisons between our results and those of the previous landmark study are worth a deeper analysis. Levinson et $\mathrm{al}^{8}$ included in their study 65 emotional expressions in both surgical and primary care encounters, and used a different methodology to characterize clinician responses to patient emotional expressions as either positive or missed opportunities. These categories were therefore evaluative (ie, good vs bad), whereas the VR-CoDES is descriptive. It has been the subsequent interpretations of the study by Levinson and colleagues that have emphasized the benefits of "empathy" specifically, but this interpretation of empathy may differ from the more limited definition provided by the VR-CoDES. This discordance is evident from the differences in rates of responses categorized as "positive" in the that study (28\%) vs responses categorized as "empathy" in ours (5\%). The low rate of empathy seen in our study also makes it more difficult to observe significant associations between empathy and outcomes. It is possible that our finding about the time-saving nature of explicit responses is reflective of what Levinson and colleagues contributed to their categorization of positive responses.

When clinician responses were explicit (vs nonexplicit), we found that patients were less likely to repeat their emotional expression, and there was a trend toward shorter length of visit. Although the association with visit length was reduced after accounting for clinicians' tendency to be more explicit toward the end of the encounter, we still estimate a 1.37 -minute shorter visit from being explicit vs nonexplicit. The corresponding finding that a patient was less likely to repeat their emotion supports the theory that this additional time is saved because the patient feels heard. It is worth considering, however, that the time may be saved because of the active listening required to make an explicit response, rather than or in addition to the fact that the patient feels heard. In either case, the advice to clinicians would be to respond explicitly, which requires mindful attention and focus, which is of practical use in teaching about communication skills.

It is interesting to note that clinician responses varied over the course of the encounter. As time passed in medical visits, clinicians were less likely to respond to patient emotional expressions by providing space and more likely to make their response explicit. These findings are perhaps not surprising, although to our knowledge they have not been previously demonstrated. In a related line of reasoning, however, one study found oncologists gave more attention to the first emotional expression raised by the patient and less attention to later concerns. ${ }^{14}$ The typical arc of a routine patient encounter allows for a greater amount of patient talk in the beginning when the clinician is gathering information and may feel less hurried, rather than toward the end when the clinician may be providing information and trying to wrap up loose ends. By reducing space and making it known to the patient that the clinician has heard the emotional expression, the clinician may be attempting to limit the conversation further. This phenomenon is important to consider when evaluating the true effect of clinician behavior on visit length because failing to account for it will distort the association between clinician response and visit length.

The results of this study should be interpreted in light of several limitations. We collected data from HIV specialty centers at 2 urban academic medical centers located in the United States, which may limit the generalizability of our results to other health care settings and populations. Because HIV has become a chronic condition and many of the patients in these clinics rely on their HIV clinicians for primary care, we believe this setting is similar to many primary encounters with medically and socially complex patients. Although all patients had HIV, we did not collect data on other comorbidities such as mood disorders (depression or anxiety), substance use disorders, or other physical health issues. These comorbidities could impact visit length as well as emotional expressions, and would be important to explore in future studies.

Our study sample comprises primarily White, female clinicians and African American, male patients. Although these demographics are reflective of the HIV epidemic overall, the sample's skewed racial and sex composition, the diversity of health professionals represented, or race-sex discordance may influence clinician communication around patient emotions. ${ }^{12,14,15}$ Finally, all patients and clinicians in this study spoke English in the encounter, and all had (as specified by inclusion criteria) been in a relationship with each other for at least 6 months. Whether emotional communication changes over time in patient-clinician relationships is not known.

Because we assessed verbal behavior through audio-recorded encounters, we were not able to evaluate nonverbal behaviors that could provide clues to patients' negative emotions and contribute to empathic responses by the clinician. The definition of an empathic response was limited to verbal behavior, as it is in many studies using the VR-CoDES. ${ }^{16,17}$ Similarly, 
the limitation of our analysis to audio-recorded interactions means that we could not know the intention of the clinician responses (e.g., whether the responses intended to be helpful or perhaps were an automatic pattern of response), nor could we ascertain the impact that the responses had on the patient's experience. Finally, our data were coded by a single experienced coder, and we did not assess intercoder reliability. Previous studies that have done so, however, have found discrepancies to be small. ${ }^{10}$

In contrast to common understanding, our study found that there was no association between the specific response of empathy and visit length. Our findings suggest that some clinician responses-such as providing space for patients to talk about their emotions-can be considered beneficial but may add time to the visit. As medical educators think about how to teach communication skills to students and as clinicians make decisions about how to allocate a fixed amount of time for their patients, it makes sense to consider that coherent explicit responses to patient emotion may save time and are considered at least as good as-if not better than-nonexplicit responses. Providing space for patients to talk about their emotions in the HIV ambulatory care setting does not save time, but may be important for other reasons.

To read or post commentaries in response to this article, go to https://www.AnnFamMed.org/content/19/6/515/tab-e-letters.

Key words: patient-clinician communication; emotions; psychological distress; empathy; primary care; office visits; time management; behavior; personalized care; practice-based research

Submitted July 1, 2020; submitted, revised, March 15, 2021; accepted April 13, 2021.

Supplemental materials: Available at https://www.AnnFamMed. org/lookup/suppl/doi:10.1370/afm.2740/-/DC1.

\section{References}

1. Wells KB, Benson MC, Hoff P. A model for teaching the brief psychosocial interview. J Med Educ. 1985;60(3):181-188.

2. Branch WT, Malik TK. Using 'windows of opportunities' in brief interviews to understand patients' concerns. JAMA. 1993;269(13): 1667-1668.
3. Suchman AL, Markakis K, Beckman HB, Frankel R. A model of empathic communication in the medical interview. JAMA. 1997; 277(8):678-682.

4. Bylund $\mathrm{CL}$, Makoul G. Examining empathy in medical encounters: an observational study using the empathic communication coding system. Health Commun. 2005;18(2):123-140.

5. Zimmermann C, Del Piccolo L, Bensing J, et al. Coding patient emotional cues and concerns in medical consultations: the Verona coding definitions of emotional sequences (VR-CoDES). Patient Educ Couns. 2011;82(2):141-148.

6. Del Piccolo L, de Haes H, Heaven C, et al. Development of the Verona coding definitions of emotional sequences to code health providers' responses (VR-CoDES-P) to patient cues and concerns. Patient Educ Couns. 2011;82(2):149-155.

7. Morse DS, Edwardsen EA, Gordon HS. Missed opportunities for interval empathy in lung cancer communication. Arch Intern Med. 2008;168(17):1853-1858.

8. Levinson W, Gorawara-Bhat R, Lamb J. A study of patient clues and physician responses in primary care and surgical settings. JAMA. 2000;284(8):1021-1027.

9. Eide $H$, Eide $T$, Rustøen $T$, Finset $A$. Patient validation of cues and concerns identified according to Verona coding definitions of emotional sequences (VR-CODES): a video- and interview-based approach. Patient Educ Couns. 2011;82(2):156-162.

10. Fletcher I, Mazzi M, Nuebling M. When coders are reliable: the application of three measures to assess inter-rater reliability/agreement with doctor-patient communication data coded with the VRCoDES. Patient Educ Couns. 2011;82(3):341-345.

11. Park J, Saha S, Han D, et al. Emotional communication in HIV care: an observational study of patients' expressed emotions and clinician response. AIDS Behav. 2019;23(10):2816-2828.

12. Park J, Beach MC, Han D, Moore RD, Korthuis PT, Saha S. Racial disparities in clinician responses to patient emotions. Patient Educ Couns. 2020;103(9):1736-1744.

13. Park J, Saha S, Han D, et al. Are clinicians' self-reported empathic concern and perspective-taking traits associated with their response to patient emotions?: Communication Studies. Patient Educ Couns. 2020;103(9):1745-1751.

14. Finset $A$, Heyn L, Ruland C. Patterns in clinicians' responses to patient emotion in cancer care. Patient Educ Couns. 2013;93(1):80-85.

15. Del Piccolo L, Mazzi MA, Goss C, Rimondini M, Zimmermann C. How emotions emerge and are dealt with in first diagnostic consultations in psychiatry. Patient Educ Couns. 2012;88(1):29-35.

16. Del Piccolo L, Mazzi MA, Mascanzoni A, et al. Factors related to the expression of emotions by early-stage breast cancer patients. Patient Educ Couns. 2019;102(10):1767-1773.

17. Schouten $B C$, Schinkel S. Emotions in primary care: are there cultural differences in the expression of cues and concerns? Patient Educ Couns. 2015;98(11):1346-1351. 